\title{
ENFERMEIRO HOSPITALAR E O STRESS
}

\author{
STRESS OF HOSPITAL NURSES
}

Estela Regina Ferraz Bianchi *

BIANCHI, E.R.F. Enfermeiro hospitalar e o stress. Rev.Esc.Enf.USP, v. 34, n.4, p. 390-4, dez. 2000.

\section{RESUMO}

Relata a comparação do nivel de stress no desempenho das atividades do enfermeiro de instituição hospitalar em unidades consideradas abertas e fechadas. Constatou-se que os enfermeiros que atuam em unidades abertas obtiveram maior nivel de stress do que aqueles que trabalham em unidades fechadas e praticamente a atuação relacionada à administração de pessoal foi considerada estressante para a totalidade de enfermeiros. E necessária a educação do enfermeiro para minimizar esses fatores estressantes.

PALAVRAS-CHAVE: Estresse. Estafa profissional. Enfermeiros. Pesquisa em enfermagem.

\section{ABSTRACT}

his study is a comparison between hospital nurses who work in "open and closed" units. In open units, patient and his family have free transit and in closed units, like critical care unit, patient and his family have limitation in transit. The results demonstrated that nurses who worked in open unit had higher stress score than nurses in closed units. For both groups, personal administration had high stress score. The nurses' education is important to improve her performance.

KEYWORDS: Stress. Burnout professional. Nurses male. Nursing research.

\section{INTRODUÇÃO}

Stress é um tema de larga abrangência e que ocupa lugar de destaque nos diversos meios de comunicação. E amplamente abordado em temas de discussão popular, assim como na literatura científica.

Stress permeia a vida do homem desde a Antiguidade - na luta com os animais para promover a sua sobrevivência, por exemplo. Com o passar dos anos, várias abordagens foram surgindo. Como pontos marcantes nesse desenvolvimento tem-se SELYE (1956) que definiu o chamado stress biológico, com a descrição da síndrome de adaptação geral (SAG). Foi chamado o "pai " da teoria do stress devido à delimitação que colocou no uso do termo stress, isto é, pode-se falar em stress desde que haja a liberação de catecolaminas, glicocorticóides e mineralocorticóides. Apesar desse grande avanço, houve a necessidade de englobar o importante papel desempenhado pelo individuo, colocando a avaliação do sujeito em relação ao estressor como peça fundamental no desencadeamento do stress.
LAZARUS; LAUNIER (1978) definem stress, no modelo interacionista, como qualquer evento que demande do ambiente externo ou interno e que taxe ou exceda as fontes de adaptação de um indivíduo ou sistema social. Tem como etapas a avaliação primária, realizada quando o individuo se confronta com o evento e o avalia como irrelevante, e não provocador de stress ou como um desfio ( positivo) ou uma ameça (negativo) e ambos desencadeadores das manifestações biológicas da SAG. Descrevem a avaliação secundária, quando o individuo avalia seus potenciais para enfrentar a situação estressante e como pode usar os mecanismos de coping.

O estudo de stress entre enfermeiros teve início por volta dos anos sessenta, quando na realidade estrangeira surgiu a preocupação com o profissional irritado, desapontado e culpado por não conseguir lidar com esses sentimentos, descritos por MENZIES (1960). Observa-se que houve um predominio de trabalhos realizados primordialmente com

\footnotetext{
Professor Associado junto ao Departamento de Enfermagem Médico-cirúrgica, Escola de Enfermagem da USP. Livre Docente em Enfermagem.
} 
enfermeiros que atuavam em unidades de terapia intensiva, pois coincidiu com o início da conquista de novos espaços e novas tecnologias por esses profissionais. Outros trabalhos de comparação entre unidades foram realizados e uma tentativa de delinear um modelo de repercussão de stress na atuação do enfermeiro. Entretanto, depara-se com a diversificação de metodologias usadas e de referenciais teórico-práticos, que ocorre nos estudos realizados no campo de stress.

No Brasil, encontram-se publicações voltadas para o stress na década de noventa, com o trabalho realizado por BIANCHI (1990) junto a enfermeiros de centro cirúrgico, SILVA; BIANCHI (1992) com enfermeiros de centro de material, CANDEIAS et al (1992) que pesquisaram o stress numa equipe de enfermagem atuante num hospital de cardiologia; CHAVES (1994) que estudou a influência do stress no trabalho do turno noturno; LAUTERT (1997) que verificou o desgaste físico e emocional entre enfermeiros; FERREIRA ( 1998) que pesquisou stress junto à equipe de enfermagem atuante em terapia intensiva; STACCIARINI (1999) que estudou stress dos enfermeiros e a proposta de um modelo. Percebe-se que há uma diversificação de abordagens, reflexo da gama de estudos existentes. Apontou-se no presente estudo algumas pesquisas realizadas, mas deve-se ressaltar que há vários artigos de atualização e temas de eventos científicos preocupados com o assunto.

Há a concordância entre os autores que ser enfermeiro é pertencer a uma profissão estressante. Após a realização do estudo junto aos enfermeiros de centro cirúrgico ficou evidente a necessidade de realização de um estudo de comparação entre as unidades de atuação dos enfermeiros. É de conhecimento, que a atuação junto ao paciente crítico é desgastante e esforços são obtidos para aprimorar cada vez mais essas áreas de atuação, surgindo os cursos de especialização. Entretanto, sabe-se também que na nossa realidade de saúde, o paciente é instável e crítico em unidades de internação onde deveria existir a condição de estabilidade do estado geral do paciente. E como fica o stress desse profissional?

Diante dessas indagações realizou-se o presente estudo, tendo como objetivos verificar o nivel de stress para os enfermeiros que atuam em unidades abertas e fechadas da instituição hospitalar e caracterizar os estressores na atuação desses enfermeiros .

\section{CASUÍSTICA E MÉTODO}

Este estudo é quantitativo, transversal e descritivo sobre a condição de stress entre os enfermeiros que trabalham em unidades hospitalares.

Os dados foram coletados junto a 116 enfermeiros que pertenciam a duas instituições hospitalares do município de São Paulo, sendo uma governamental e outra beneficente. Essa amostra representou 38,3\% da população total. Houve autorização dos comitês de ética e dos enfermeiros envolvidos.

A coleta de dados foi realizada utilizando-se um questionário, adaptado do trabalho anterior BIANCHI (1990). Houve a revisão por juizes para retirar a especificidade da atuação em centro cirúrgico e realizado um pré-teste.

O questionário consta de dados de identificação e um levantamento de atividades estressantes para o enfermeiro usando a escala de Likert variando de 1 como "pouco desgastante"; valor médio 4 e valor 7 para "altamente desgastante". O valor 0 foi reservado para assinalar quando não realizasse a atividade.

Os questionários foram distribuídos pessoalmente a cada enfermeiro, esclarecida a finalidade de estudo e sua participação, e a devolução foi feita em local determinado.

Os dados foram analisados mediante a padronização do escore para cada enfermeiro e para cada área de atuação usada para o presente estudo.

As atividades estressantes foram englobadas nas áreas: A- Relacionamento com outras unidades e supervisores; B- Atividades relacionadas ao funcionamento adequado da unidade; $\mathrm{C}$ - Atividades relacionadas à administração de pessoal; DAssistência de enfermagem prestada ao paciente; ECoordenação das atividades da unidade; F Condições de trabalho para o desempenho das atividades do enfermeiro .

A classificação das unidades de atuação do enfermeiro em unidade aberta e fechada foi realizada levando-se em consideração o fluxo de pacientes e familiares. Considerou-se como unidade aberta a unidade de internação, unidade de pronto atendimento, comissão de controle de infecção e unidade de internação em maternidade.Como fechada foram englobadas as unidades de terapia intensiva, centro cirúrgico, centro de material, centro obstétrico, transplante de órgãos, endoscopia, berçário, hemodinâmica e hemodiálise.

\section{RESULTADOS E DISCUSSÃO}

\section{1- Caracterização da população}

A população do estudo reflete a constituição da população de enfermeiros no Brasil, isto é, eminentemente feminina $(93,2 \%)$.

Na faixa etária, houve predominância de 20 a 40 anos, perfazendo um total de $81,4 \%$.

Na distribuição de cargos ocupados houve uma predominância de enfermeiros assistenciais $(83,1 \%)$; chefes $(10,1 \%)$ e supervisores $(6,8 \%)$. 
Os enfermeiros pertencentes à unidade aberta $(56,0 \%)$ foram em maior número do que os de unidade fechada $(44,0 \%)$, com um tempo de formado de até
10 anos $(59,3 \%)$ e tendo freqüentado algum curso de pós-graduação $(50,8 \%)$.

\section{2 -Escore padronizado para cada tipo de unidade hospitalar}

Gráfico 1. Distribuição dos escores padronizados de stress para unidades abertas e fechadas de acordo com cada área de atuação do enfermeiro. São Paulo, 1997.

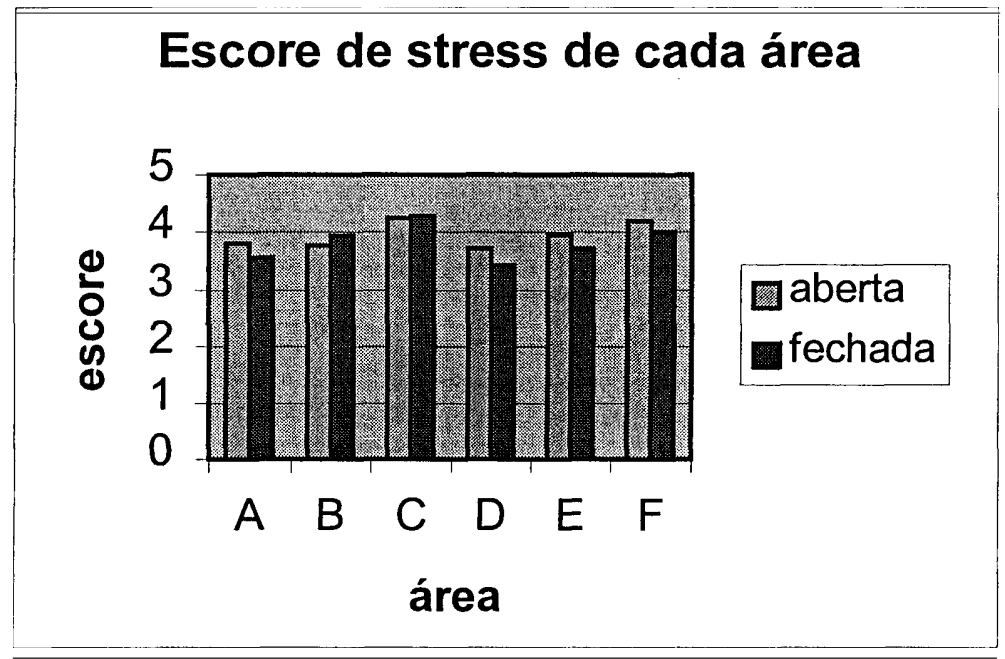

A distribuição dos escores padronizados entre as unidades ditas abertas e fechadas foi praticamente homogênea. Os escores mais altos para as unidades ABERTAS foram para as áreas: A (Relacionamento com outras unidades e supervisores); D (Assistência de enfermagem prestada ao paciente); E (Coordenação das atividades na unidade); F (Condições de trabalho para o desempenho das atividades do enfermeiro). Para as unidades FECHADAS o escore mais alto do que as unidades abertas foi somente na área B (Atividades relacionadas ao funcionamento adequado da unidade). Praticamente não houve diferença entre as unidades abertas e fechadas na área C (Atividades relacionadas à administração de pessoal).

Retomando o conceito de LAZARUS; LAUNIER (1978), stress é avaliado como desafio ou como ameaça e as repercussões são detectadas nos sistemas orgânicos, psicológico, social e espiritual e são dependentes dos mecanismos de coping disponiveis ao indivíduo.

Sendo assim, os fatores externos como o trabalho, a família, o ambiente entre outros podem influenciar a percepção de stress assim como os fatores internos e entre eles as emoções, experiência anterior, crenças e valores.

Percebeu-se que houve enfermeiros que apresentaram nivel baixo de stress com escore abaixo de 2,0 e também houve aqueles com alto nivel como os que obtiveram escore maior que 5,0.

Esses dados nos levam a ponderar que a instituição hospitalar é responsável pela diminuição dos fatores externos, concernentes às condições detrabalho, possibilitando ao enfermeiro o desenvolvimento de suas potencialidades e preservando a 392 integridade fisica e emocional para lidar com os problemas dos pacientes e seus familiares.

APPELBAUM (1981) relata que a organização piramidal da instituição hospitalar é responsável pelas pressões que os profissionais de saúde sofrem, excluindo os médicos que geralmente não pertencem à instituição e somente usam o ambiente para o desenvolvimento de suas atividades, levando ao conflito entre comportamento médico e o comportamento dos demais profissionais da saúde. Acrescenta ainda, que o ambiente da instituição hospitalar tem mais problemas comportamentais do que técnicos.

Em relação ao stress, a instituição hospitalar deve proporcionar discussões entre os grupos para o conhecimento das bases teóricas sobre o assunto e propiciar um momento de revelação dos conflitos e de discussão de possibilidades de diminuição de stress. Uma proposta por nós testada é a inoculação de stress (BIANCHI, 1990) e que defende a aquisição de conhecimento sobre stress como mecanismo de coping efetivo para as pessoas enfrentarem seus estressores com menor repercussão para o indivíduo e para a organização.

Destacando-se cada área de atuação do enfermeiro, obteve-se que na área A - Relacionamento com outras unidades e supervisores, constituída de nove situações, para o enfermeiro de unidade ABERTA houve maior escore nas atividades: relacionamento com outras unidades; relaciona-mento com a farmácia, com a manutenção, com o setor de alta 
e admissão de pacientes, comunicação com supervisores de enfermagem e com a administração superior. Os de unidade FECHADA obtiveram maior índice somente no relacionamento com almoxarifado. O relacionamento com centro de material e centro cirúrgico obtiveram praticamente os mesmos escores.

Para a área B - Atividades relacionadas ao funcionamento adequado da unidade (seis situações), os enfermeiros da unidade FECHADA manifestaram maior nivel de stress nas atividades: previsão de material a ser usado; reposição de material; controle de material usado; levantamento de quantidade de material existente na unidade. Para os enfermeiros de unidade $\boldsymbol{A B E R T A}$ o maior índice foi em relação à solicitação de revisão e conserto de equipamentos. O escore foi igual em relação ao controle de equipamentos.

$\mathrm{Na}$ área $\mathrm{C}$ - Atividades relacionadas à administração de pessoal ( seis situações), houve maior indice de stress para os enfermeiros de unidade $\boldsymbol{A B E R T A}$ quanto a: controlar a equipe de enfermagem ; realizar a distribuição de funcionários; supervisionar as atividades da equipe; elaborar escala mensal de funcionários. Para os de unidade FECHADA foram: realizar treinamento; avaliar o desempenho do funcionário. Cabe destacar que essa área obteve os maiores indices para os enfermeiros independentemente de sua unidade de atuação.

Para a área D - Assistência de enfermagem prestada ao paciente (quinze situações), os enfermeiros de unidade $\boldsymbol{A B E R T A}$ obtiveram maior indice de stress nas seguintes atividades: admitir o paciente na unidade; fazer exame físico do paciente; avaliar condições fisicas do paciente; atender às necessidades do paciente; atender às necessidades dos familiares; orientar o paciente para o auto-cuidado; orientar os familiares para cuidar do paciente; supervisionar o cuidado de enfermagem prestado; orientar para a alta do paciente; prestar cuidados de enfermagem; atender os familiares de pacientes criticos; enfrentar a morte do paciente; orientar familiares de paciente crítico. Para os de unidade FECHADA os escores mais altos foram: prescrever cuidados de enfermagem e atender às emergências na unidade.

$\mathrm{Na}$ área E - Coordenação das atividades da unidade (oito situações), os enfermeiros de unidade ABERTA relataram maior nível de stress em relação a: controlar a qualidade do cuidado; coordenar as atividades da unidade; realizar discussão de caso com funcionários; realizar discussão de caso com equipe multiprofissional; elaborar rotinas, normas e procedimentos; atualizar rotinas, normas e procedimentos. Os que atuam em unidade FECHADA obtiveram maior indice quanto a elaborar o relatório mensal da unidade. A definição das funções do enfermeiro obteve escore semelhante entre os enfermeiros, com nivel médio de stress.

Para a área $\mathrm{F}$ - Condições de trabalho para o desempenho das atividades do enfermeiro (seis situações), Os enfermeiros de unidade $\boldsymbol{A B E R T A}$ relataram maior indice de stress quanto a: participar de reuniões do Departamento de Enfermagem; participar de comissões da instituição; participar de eventos científicos; ambiente físico da unidade; realizar tarefas burocráticas e realizar tarefas com tempo minimo disponivel.

No presente estudo, os enfermeiros das unidades $\boldsymbol{A B E R T A S}$ relataram alto nivel de stress quanto ao relacionamento com outras unidades e supervisores, assistência de enfermagem prestada ao paciente, coordenação das atividades na unidade e condições de trabalho para o desempenho das atividades do enfermeiro. Das seis áreas classificadas no estudo, os enfermeiros de unidade aberta obtiveram maior escore de stress em quatro delas, e uma variação do escore padronizado de stress de 2,32 a 5,63. Evidencia-se uma condição de trabalho que favorece o surgimento de stress e até de burnout.

ANDERSON et al (1988) relataram que os enfermeiros que trabalhavam em unidade médicocirúrgica obtiveram maior nivel de stress do que os de unidade de terapia intensiva. As categorias de estressores pesquisadas por esses autores foram: assistência ao paciente; administração da unidade; relacionamento interpessoal; conhecimentos e habilidades; ambiente físico da unidade; eventos da vida e recompensas administrativas. A categoria considerada mais estressante para os enfermeiros da unidade médico-cirúrgica foi a administração da unidade enquanto que para os de unidade de terapia intensiva foi o relacionamento interpessoal.

Para as unidades fechadas, o maior escore de stress foi quanto às atividades relacionadas ao funcionamento adequado da unidade. Isto é até esperado pela alta tecnologia empregada nessas unidades. No presente estudo, as unidades englobadas são centro cirúrgico, centro de material, unidade de terapia intensiva, transplante, berçário, hemodinâmica e hemodiálise. A previsão, a provisão e ainda a prontidão na obtenção de materiais e equipamentos além do controle desses artefatos é quase que imprescindivel para a realização da assistência prestada ao paciente e a sua falta é mais gritante nessas unidades.

LAUTERT (1997) ao estudar o desgaste emocional de enfermeiros de dois hospitais universitários de Porto Alegre, utilizando o referencial de Maslach e Jackson, obteve que as enfermeiras de unidades de internação encontram-se com um desgaste emocional e a autora aponta a correlação com sobrecarga de trabalho, tarefas repetitivas e monótonas e volume de atividades burocráticas existente levandoas à falta de controle das atividades realizadas, perda de energia, fadiga e esgotamento.

Em relação à administração de pessoal praticamente não houve diferença entre os enfermeiros de unidades abertas e fechadas e com a média de escore padronizado de 4,29. Podemos inferir que as atividades englobadas nessa área, isto é, o 
controle, supervisão, treinamento e avaliação da equipe de enfermagem é uma área estressante na atuação do enfermeiro. Sabe-se que o relacionamento pessoal é dificil de ser conseguido e na administração de pessoal, o relacionamento faz parte dessa atuação.

Isto nos faz refletir sobre a autonomia e poder de decisão do enfermeiro. Sendo essa área uma relação direta com a tomada de decisão em niveis hierárquicos superiores e de demanda de poder, será que o enfermeiro está sendo consciente desse poder de conseguir o pessoal necessário e as condições adequadas de trabalho.

Percebe-se que em algumas instituições o poder do enfermeiro é conseguido e defendido. Em outras, o poder da organização é maior, e não é evidente mas sub-liminarmente há outros interesses, como o lucro e a mercantilização da medicina, como nos alerta PITTA ( 1991) .

Deve-se ressaltar o papel importante que tem cada elemento envolvido nesse contexto, isto é, do enfermeiro, da instituição hospitalar e do órgão formador desse profissional.

O enfermeiro é responsável, cada vez mais, por sua educação, seja em cursos de pós-graduação como em reciclagem e ele deve mostrar o seu potencial e interesse para a instituição. Deve ainda ter consciência de que a subjetividade diante de um evento é indispensável na avaliação de um evento como estressante ou não, podendo utilizar de mecanismos de coping individual, como a obtenção de conhecimentos, horário de lazer e distração, meditação, formas de enfrentamento entre outros, para atenuar ou até modificar a avaliação proposta, promovendo uma melhoria da qualidade de vida desse profissional.

A instituição deve investir no aprimoramento e reciclagem do enfermeiro, assim como promover as condições básicas para a atuação adequada desse profissional. Os mecanismos de coping organizacional, tais como reconhecimento do trabalho executado, incentivo na remuneração e na participação do enfermeiro, supervisão com orientação, participação na tomada de decisão entre outros, podem constituir fator de alivio de stress, proporcionando um ambiente favorável de trabalho erevertendo para a qualidade de assistência prestada ao paciente e aos familiares.

O órgão formador é responsável por uma atuação respaldada em conhecimentos científicos e que proporcionem ao enfermeiro uma base para o desempenho com menor stress. Algumas propostas de aconselhamento de alunos têm sido veiculadas, como uma maneira de aliviar o stress do aluno e possibilitar o seu aprendizado de mecanismos que utilizará em todo o transcorrer de sua vida profissional.

\section{CONSIDERAÇÕES FINAIS}

Este estudo mostrou que o stress está presente na atuação do enfermeiro em instituições hospitalares. junto ao paciente crítico são estressados não é sustentada neste trabalho. Os enfermeiros de unidades abertas apresentaram maior índice de stress em relação àqueles que atuavam em unidades fechadas.

Com esta constatação há necessidade de discutir as condições de trabalho do enfermeiro, independente de sua área de atuação, como uma profissão estressante e que deve ser reconhecida como tal. Há necessidade de investimento do enfermeiro, preocupado com sua atuação, da instituição hospitalar e dos cursos de graduação e pós-graduação.

O sucesso da prestação da assistência ao paciente efamília se faz com profissionais que estejam preparados globalmente, isto é, no sentido de conhecimentos, emoções e atuação com estrutura adequada.

\section{REFERÊNCIAS BIBLIOGRÁFICAS}

ANDERSON, M. et al. Changes in management stressors on ICU nurses. Dimens. Crit. Care Nurs., v.9, n.2, p.111-7, 1988.

APPELBAUM, S.H. Stress management for health care professions. Rockville, Aspen , 1981. cap.5, p.109- 47 : The impact of stress on nurses and other health care professionals.

BIANCHI, E.R.F. Estresse em enfermagem: análise da atuação do enfermeiro de centro cirúrgico. São Paulo, 113p., 1990. Tese (Doutorado) - Escola de Enfermagem, Universidade de São Paulo.

CANDEIAS, N.M.F. et al. Stress em atendentes de enfermagem. Rev.Bras.Saúde Ocup., v.20, n.75, p.38-44, 1992.

CHAVES, E.C. Stress e trabalho do enfermeiro: a influência de caracteristicas individuais no ajustamento e tolerância ao turno noturno. São Paulo, 1994. 130 p. Tese (Doutorado) Instituto de Psicologia, Universidade de São Paulo.

FERREIRA, F.G. Desvendando o estresse da equipe de enfermagem em terapia intensiva. São Paulo, 1998. 136p. Dissertação (Mestrado) - Escola de Enfermagem, Universidade de São Paulo.

LAUTERT, L. O desgaste profissional: estudo empírico com enfermeiras que trabalham em hospitais. Rev. Gaúcha Enf., v.18, n.2, p.133- 44, 1997.

LAZARUS, R.S.; LAUNIER, S. Stress related transaction between person and environment. In: DERVIN, L.A.; LEWIS, M. Perspectives in international psychology. New York, Plenum, 1978. p.287-327.

MENZIES, I.E.P. Nurses under stress. Int. Nurs. Review,v.7, n.6, p.9-16, 1960 .

PITTA, A.M. Hospital: dor e morte como oficio. 2.ed. São Paulo, Hucitec, 1991.

SELYE, H. The stress of life. New York, Mc Graw-Hill, 1956.

SILVA, A.; BIANCHI,E.R.F. Estresse ocupacional da enfermeira de centro de material. Rev. Esc. Enf. USP, v.26, n.1, p.65-74, 1992 .

STACCIARINI, J.M.R. Estresse ocupacional, estilos de pensamento e coping - na satisfação, mal estar físico e psicológico em enfermeiros. Brasília, 1999. 304p. Tese (Doutorado) - Instituto de Psicologia, Universidade de Brasília. 